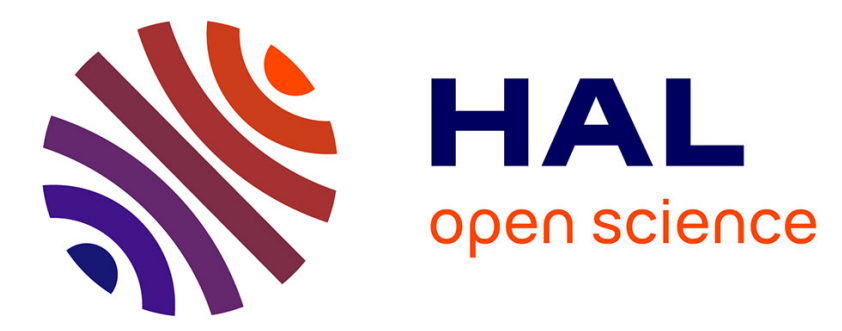

\title{
The effect of bottom roughness in two-layer flows down a slope
}

\author{
Maria-Eletta Negretti, David Z Zhu, Gerhard H Jirka
}

\section{To cite this version:}

Maria-Eletta Negretti, David Z Zhu, Gerhard H Jirka. The effect of bottom roughness in twolayer flows down a slope. Dynamics of Atmospheres and Oceans, 2008, 45 (1-2), pp.46-68. 10.1016/j.dynatmoce.2008.02.001 . hal-01904477

\section{HAL Id: hal-01904477 \\ https://hal.science/hal-01904477}

Submitted on 22 Jan 2020

HAL is a multi-disciplinary open access archive for the deposit and dissemination of scientific research documents, whether they are published or not. The documents may come from teaching and research institutions in France or abroad, or from public or private research centers.
L'archive ouverte pluridisciplinaire HAL, est destinée au dépôt et à la diffusion de documents scientifiques de niveau recherche, publiés ou non, émanant des établissements d'enseignement et de recherche français ou étrangers, des laboratoires publics ou privés. 


\title{
The effect of bottom roughness in two-layer flows down a slope
}

\author{
M.Eletta Negretti ${ }^{\text {a,* }}$, David Z. Zhu ${ }^{\text {b }}$, Gerhard H. Jirka ${ }^{a}$ \\ a Institute for Hydromechanics, University of Karlsruhe, Kaiserstr. 12, D-76131 Karlsruhe, Germany \\ ${ }^{\mathrm{b}}$ Department of Civil and Environmental Engineering, University of Alberta, \\ T6G2W2 Edmonton, Alberta, Canada
}

\begin{abstract}
Laboratory experiments were conducted to study the effect of locally enhanced bottom roughness in a density-stratified two-layer flow down a slope. Three bottom roughness configurations were investigated, a smooth bed and two artificially roughened beds, employing sparse and dense roughness elements, respectively. The bottom roughness elements have shown to affect differently the generation and collapse mechanisms of large-scale two-dimensional structures observed at the interface between the two layers: dense bottom roughness inhibits the generation of large-scale structures while sparse bottom roughness inhibits their collapsing mechanism. Both bottom roughness configurations cause a reduction of the size of the large structures at the interface as compared to the smooth case. Two main sources of entrainment have been identified, namely the observed large-scale structures at the interface and enhanced bottom roughness. Sparse bottom roughness gives the lowest entrainment coefficients among the three cases due to the low interaction between the bottom boundary layer and the interface.
\end{abstract}

Keywords: Stratified flows; Bottom roughness; Topographic effects; Interfacial waves; Entrainment; Vorticity

\section{Introduction}

Two-layer density stratified flows are commonly observed in the oceans, in lakes and in the atmosphere. Examples of such flows in the oceanographic context include the two-layer flow in the Strait of Gibraltar exchanging the water mass between the Mediterranean sea and the North

\footnotetext{
* Corresponding author. Tel.: +49 721608 3538; fax: +49 7216082202.

E-mail address: negretti@ifh.uka.de (M.Eletta Negretti).
} 
Atlantic (Armi and Farmer, 1986) and that in the Faroe Bank channel connecting the GreenlandIceland-Norwegian Seas to the North Atlantic (Hansen and Osterhus, 2000). The two-layer flows in these channels play a crucial role in determining the distribution of water masses in the global ocean. Two-layer flows are also important in many hydraulic/environmental applications (e.g. lakes and reservoirs with interconnections and side arms) and in metereology (e.g. downslope windstorms in mountains areas). At the interface of two-layer flows small-scale instabilities, like Kelvin-Helmholtz instabilities as well as large-scale instabilities have been observed (Sherwin and Turrell, 2005; Negretti et al., 2007). Understanding of these flow processes is crucial in predicting vertical transport of heat, oxygen, nutrients and pollutants in inland water bodies, oceans, and the atmosphere. In metereology for example, it has been estimated that instability and mixing causes a 50\% increase in the drag of atmospheric flows and thus the study of shear instabilities in these flows is important also for atmospheric flow modeling and weather forecasting (Afanasyev and Peltier, 2001).

The effect of bottom roughness on the entrainment processes has been studied by Hebbert et al. (1979), Dallimore et al. (2001), Fernandez and Imberger (1999). Other studies on two-layer stably-stratified flows considering the effects of bottom roughness have mostly been devoted to investigate how the bottom generated turbulence affects the two-layer hydraulics (Zhu and Lawrence, 1998; Zaremba et al., 2003; Gu and Lawrence, 2005). A general review of past studies on these investigations not including the effect of rotation is given in the following.

A series of papers by Armi and Farmer in the 1980s (Farmer and Armi, 1986) analyzed the hydraulics of two-layer flows over a smooth bottom topography. Pratt (1987) reported that due to frictional effects, the location of a control, located originally at the crest of a bottom sill, could be shifted away from the crest point. Zhu and Lawrence (1998) extended the two-layer hydraulic theory by incorporating the non-hydrostatic pressure distribution due to streamline curvature and friction. Anati et al. (1997) examined the relative importance of frictional and inertial forces in exchange flows in constant-width channels. Zaremba et al. (2003) developed a numerical model to study effects of friction on the steady stratified exchange flow which revealed three viscous flow regimes for a converging-diverging connecting channel and further three flow regimes when a sill is introduced (see also the numerical studies of Assaf and Hecht, 1974; Og ${ }^{2} u z$ et al., 1990). More recently, Gu and Lawrence (2005) developed a one-dimensional analytical solution to the fully nonlinear problem of two-layer frictional exchange flow within the context of internal hydraulics in a rectangular channel of constant width.

Studies on the interaction between the bottom generated turbulence and the sheared interface include the numerical study of Skyllingstad and Wijesekera (2004), who used a three-dimensional large-eddy simulation (LES) model to examine how uniformly stratified flow interacts with bottom obstacles in the coastal ocean. Thorpe (1983) performed experiments of linearly stratified flows down a slope over a rough floor, where the roughness elements were represented by square bars set at regular intervals, studying both the configurations of k-type and d-type as defined in Perry et al. (1969). In his experimental study, Thorpe (1983) observed the generation of radiating internal gravity waves at the initial stage of the flow and reported turbulent mixing behind the bars due to flow separation but low interaction with the interface between the two layers. He showed that this turbulent layer spread vertically less rapidly than the internal waves and that the rate of spread depends on the separation of the bars.

Negretti et al. (2007) showed that in a two-layer stably-stratified flow with pulsating barotropic flow over smooth topography, large-scale structures are generated at the interface between the two layers. Similar structures have been also observed previously by Morin et al. (2004) and Fouli (2006). Negretti et al. (2007) explained these large-scale structures as plunging density currents 
in the lower layer triggered by the barotropic oscillation. They observed that these structures were generated with roughly the same period as the barotropic oscillation.

Continuing the work in Negretti et al. (2007), this paper is aimed at investigating the effect of enhanced bottom friction on the generation and growth mechanism of these surge-like structures and the related entrainment and mixing processes. Experiments are conducted employing two enhanced bottom roughness strategies. Following the definition of Perry et al. (1969), the first strategy is of the d-type, adopting sparse, coarse but tall bottom roughness elements above the sill, while the second strategy corresponds to the k-type, employing dense fine short bottom roughness elements. These experiments will allow us to examine the effect of enhanced bottom friction and both baroclinic and barotropic vorticity generation. Thus the results presented in this paper will deliver novel contributions more representative for natural flow conditions. In particular, the understanding of the flow interaction between the bottom generated turbulence (influenced by bottom friction) and interfacial two-dimensional surge-structures (triggered by the unsteady barotropic flow) will help to develop control methods for influencing the circulation of nutrients and pollutants in inland and coastal waters and in the atmosphere. These flow phenomena are commonly observed in nature as demonstrated by the field observations of Scinocca and Peltier (1989) and Sherwin and Turrell (2005).

The paper is organized as follows. In Section 2, the experimental design is described. A brief qualitative description of the flow is given in Section 3. In Section 4 the different mechanisms of vorticity production are discussed and specific parameters are defined. Section 5 gives an estimation of the bottom roughness coefficients through comparison to two-layer hydraulic theory. In Section 6 the experimental results are presented and discussed. Section 7 summarizes the paper.

\section{Experiments}

Experiments were conducted in a $12 \mathrm{~m}$ long and $0.6 \mathrm{~m}$ wide basin shown in Fig. 1, which is divided in the middle into two basins, and connected by a channel of reduced width $(14.5 \mathrm{~cm})$. A bottom sill has been placed in the channel. The sill shape is described by: $z(x)=h_{\mathrm{s}} \cos ^{2}(\pi x /(2 L))$, for $-40<x<0$, and $z(x)=h_{\mathrm{s}}-x / 5$, for $0<x<100$, where $h_{\mathrm{s}}=20 \mathrm{~cm}$ is the height of the sill crest and $L=100 \mathrm{~cm}$ is the length of the sill on the right hand side of the sill crest (see Fig. 1). The tank was first filled with fresh water to the desired depth $H=40 \mathrm{~cm}$. A plexiglas barrier was then placed at the sill crest to divide the tank into two reservoirs. A density difference
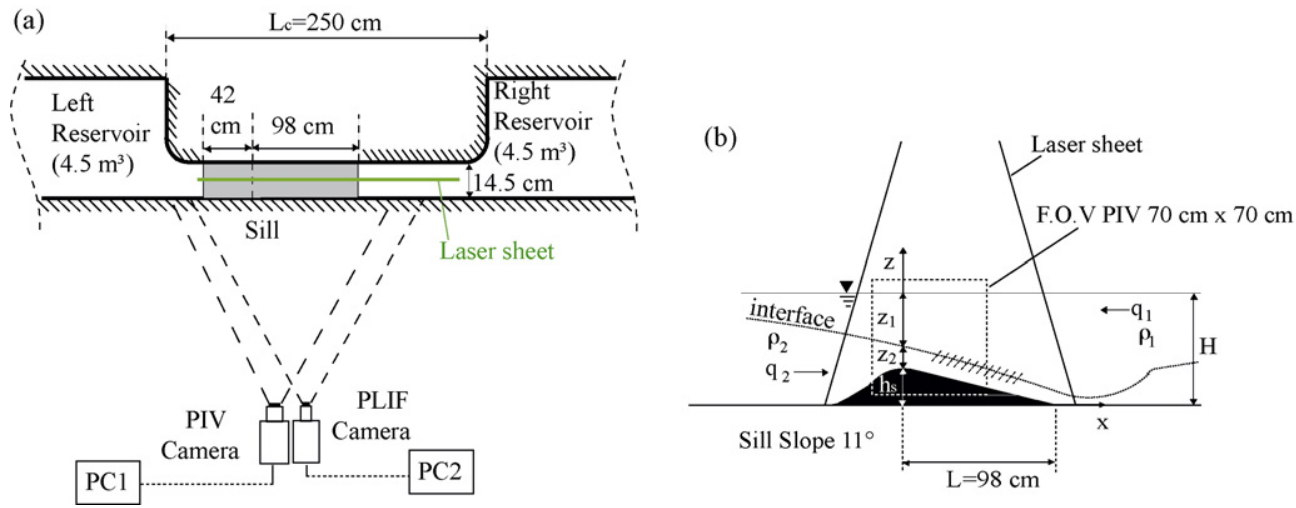

Fig. 1. Sketch of the experimental set-up. (a) Plan view and (b) side view. 
Table 1

Details of the performed experiments

\begin{tabular}{llll}
\hline Experiment properties & Smooth & Sparse & Dense \\
\hline$g^{\prime}\left(\mathrm{cm} \mathrm{s}^{-2}\right)$ & 4.47 & 4.1 & 3.97 \\
Bottom configuration & Smooth & Sparse & Dense \\
Mean height of roughness elements $(\mathrm{cm})$ & - & 3.5 & 1.6 \\
Mean spacing $(\mathrm{cm})$ & - & 3.0 & 0.1 \\
Equivalent sill height, $h^{\prime} H(\mathrm{~cm})$ & 20.0 & 20.4 & 20.7 \\
Roughness coefficient, $f_{\mathrm{w}}$ & 0.01 & 0.024 & 0.018
\end{tabular}

$g^{\prime}$ is the reduced gravitational acceleration, $h^{\prime}$ is the equivalent sill height. The roughness coefficient $f_{\mathrm{w}}$ was calculated using Eq. (13).

$\Delta \rho / \rho \approx 0.4 \%$ was produced by adding salt to the water in the left reservoir. The density of the salty water was measured with a density-meter (Anton Paar, DMA 5000). To minimize effects of wave reflections at the end of the reservoirs, two grid plates were built at each end of both reservoir (see Section 3 for a qualitative description of the flow).

Measurements of velocity and concentration fields were obtained using Particle Image Velocimetry (PIV) and Planar Laser induced Fluorescence (PLIF) techniques, respectively. Essential details are given in the following.

Polyamide particles with a mean diameter of $200 \mu \mathrm{m}$ and a density of $1.061 \mathrm{~g} / \mathrm{cm}^{3}$, were added in both reservoirs as tracer particles for the velocity measurements with PIV. A $10 \mathrm{~W}$ Argon-Ion laser operating in multimode $\left(\lambda_{1}=488 \mathrm{~nm}, \lambda_{2}=514 \mathrm{~nm}\right)$ was used as continuous light source. The beam was transmitted through a fiber optic cable to a line generator with spherical lenses. Images of roughly $70 \mathrm{~cm} \times 55 \mathrm{~cm}$ were grabbed with a CCD camera $(1024 \times 1024$ pixels $)$, at a frame rate of $10 \mathrm{~Hz}$. The raw image pairs were then processed using a PIV cross-correlation algorithm (software package DaVis, LaVision) to compute the velocity fields, starting with an interrogation window of $32 \times 32$ pixels and a final window size of $16 \times 16$ pixels with $50 \%$ overlap. Each vector of the resulting vector field represents an area of roughly $0.6 \mathrm{~cm} \times 0.6 \mathrm{~cm}$. Given the velocities encountered in the experiments, the error for the instantaneous velocity is approximately $4 \%$.

In concentration measurements Rhodamine $6 \mathrm{G}$ was used as dye and was added to the salt water. Its concentration was kept under $70 \mu \mathrm{g} / \mathrm{l}$ to assure minimal attenuation (Daviero et al., 2001). Images of $70 \mathrm{~cm} \times 55 \mathrm{~cm}$ were grabbed simultaneously with the PIV images with a CCD camera $(1280 \times 1024$ pixels $)$ at a frame rate of $10 \mathrm{~Hz}$.

Table 1 lists the experimental conditions. The smooth case was conducted using a smooth bottom, while the sparse and dense cases were performed with two different bottom roughness configurations. In the sparse roughness case, screw anchors were used (see Fig. 2(a)), with sparse spacing of coarse, tall roughness elements. This bottom roughness is similar to d-type (Perry et al., 1969). In the dense roughness case, artificial turf was used (see Fig. 2(b)), with a dense close spacing of fine, short roughness elements. This bottom roughness is similar to k-type (Perry et al., 1969).

\section{Qualitative description of the flow}

For a purely baroclinic exchange flow the net flow rate should be zero but as outlined in Negretti et al. (2007), with the experimental set-up described above, an additional unsteady barotropic flow component is superimposed on the baroclinic exchange flow. This is clearly shown in Fig. 3 , 
Top view

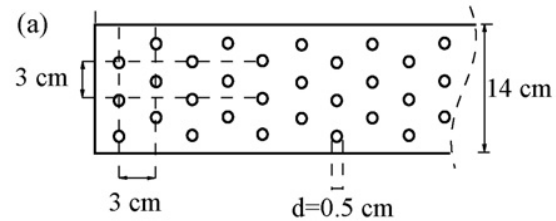

Side view

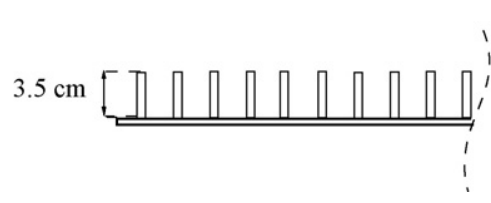

(b)

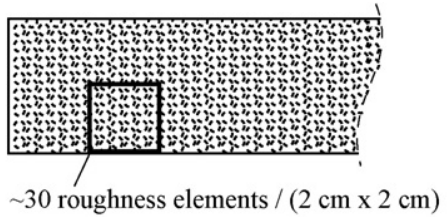

Fig. 2. Sketch of the roughness used in the rough experiments. (a) The sparse roughness case with sparse spacing of coarse, tall roughness elements (screw anchors). (b) The dense roughness case with a dense close spacing of fine, short roughness elements (artificial turf).

where the net flow rate time series at the sill crest for each experiment are plotted, normalized with the flow rate scale $q_{\text {ref }}=H \sqrt{g^{\prime} H}$, showing that it oscillates periodically around zero. These normalized net flow rates also give the ratio between the relative magnitudes of the baroclinic exchange currents $\left(q_{\mathrm{ref}}\right)$ and the superimposed barotropic oscillation $\left(\int_{h}^{H} u \mathrm{~d} z\right)$. Fig. 3 shows that the barotropic contribution is much smaller (about $8-10 \%$ for all the experiments) than the baroclinic contribution. Also, the barotropic oscillation period is smaller than the transit time of the flow across the region, which can be estimated with the internal seiche period $T_{\mathrm{S}}=2 L_{\mathrm{B}} / \mathrm{c}$, with $L_{\mathrm{B}}$ being the total basin length and $c=\sqrt{g^{\prime} z_{1} z_{2} /\left(z_{1}+z_{2}\right)}$ the propagation speed of the internal interfacial wave, with $z_{1}$ and $z_{2}$ being the water depth in the upper and lower layer, respectively (cf. Fig. 1). With a total length of $L_{\mathrm{B}}=12 \mathrm{~m}$ this gives periods of 359,375 and $381 \mathrm{~s}$ for the smooth, sparse and dense cases, respectively. The flow development at the interface for the three experiments is presented in Fig. 4 by a series of images taken during the maximal exchange regime. In the smooth case the flow is characterized by the generation of two-dimensional structures, generated at the interface due to the superimposed barotropic flow (Negretti et al., 2007) leading to large billow-like structures. The interfacial fronts pass right to the sill crest (Fig. 4(a), first and
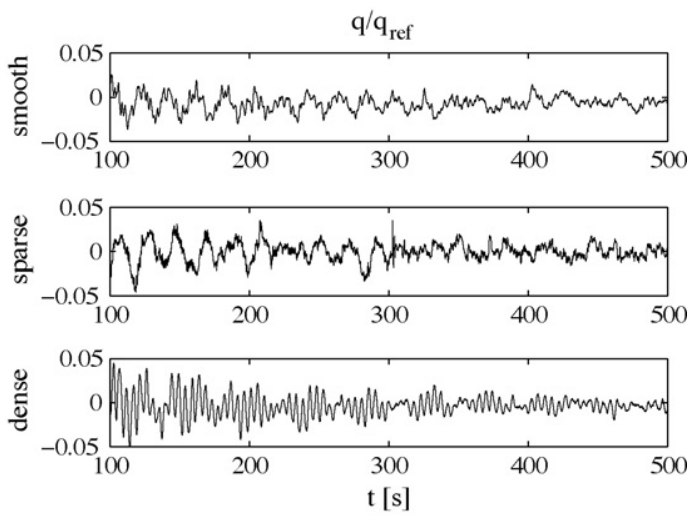

Fig. 3. Time series of the normalized net flow rate at the sill crest showing to oscillate around a zero value. This results in an unsteady barotropic flow component on the baroclinic exchange flow. 
(a)
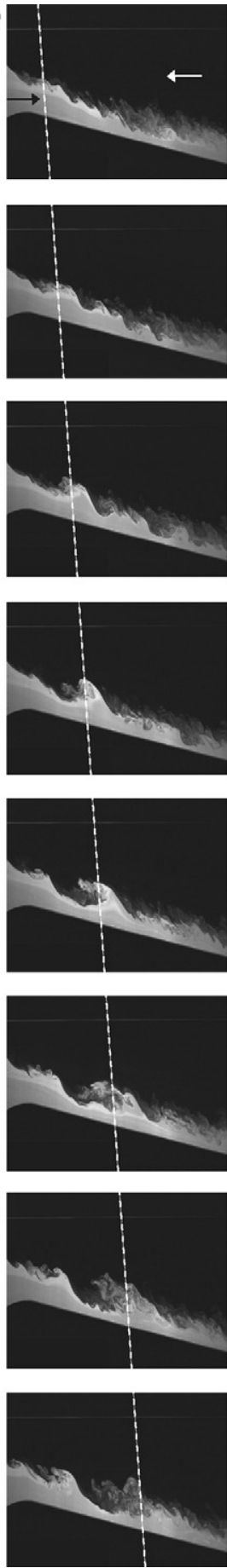

(b)
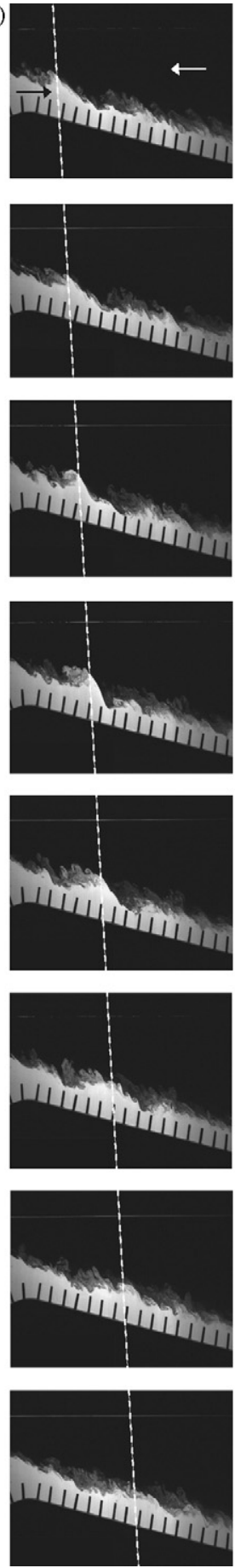
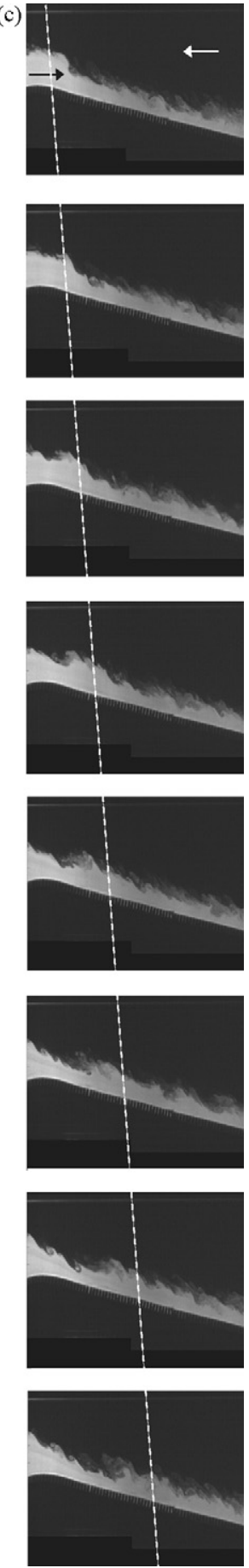

Fig. 4. Series of instantaneous images of the smooth case (a), the sparse roughness case (b) and the dense roughness case (c) with dye (Rhodamine 6G) mixed in the lower layer (salt water). The time lag between the images is $2 \mathrm{~s}$. The image size is $55 \mathrm{~cm}$ (vertical) $\times 70 \mathrm{~cm}$ (horizontal). Dashed lines follow the development of the large-scale interfacial structures. 
second image) and the flow in the lower layer begins to accelerate down the sill. In the third and fourth images, the growth of the large structure can be clearly observed, while in the following image, it starts to collapse. In this smooth case, the wake behind the surge-like flow can fully develop resulting in significant entrainment. This behavior is periodic and the phenomenon starts again from the beginning (Fig. 4(a), image 8) (Negretti et al., 2007).

The dense roughness case shows a different behavior (see Fig. 4(c)): the increase in the water depth of the lower layer is still present at the interface as a result of the pulsating barotropic flow. But the growth and subsequent roll-up mechanisms of the structures can not be observed anymore, because of the enhanced turbulence dissipation due to friction. Without roll-up of the two-dimensional structures, no wake zone is generated. However, the turbulent fluctuations are increased in this case as is discussed more in detail further below.

The sparse roughness case (see Fig. 4(b)) lies in between the behavior of the smooth and the dense roughness case: it behaves like the smooth case in the initial accelerating region near the sill crest (images 1 to 4 in Fig. 4(b)), where a growing two-dimensional structure can be observed. However, in this case, the collapse mechanism does not occur and therefore, no wake region is generated in the lee of the surges. The sparse distributed bottom roughness with tall roughness elements inhibits the roll-up mechanism of the large-scale structures when they reach a certain size and interact with the roughness elements.

\section{Vorticity production}

The definition diagram for vorticity generation in a gradually evolving two-layer flow over downslope is given in Fig. 5. Quantities in the upper layer are denoted with the index 1 while quantities in the lower layer with the index 2. In such a flow, different mechanisms are responsible for vorticity production: (1) the vortex stretching due to the variation in height of the channel. (2) The baroclinic generation of vorticity, given by the inclination of the interface. (3) The bottom generated turbulence due to the enhanced bottom roughness. Depending upon the intensity of the interaction between the bottom boundary layer and the interface between the two layers, this results possibly in an accelerated dissipation process of the turbulence produced at the interface. In the following, some parameters are defined, which can express the ratio between the magnitude of

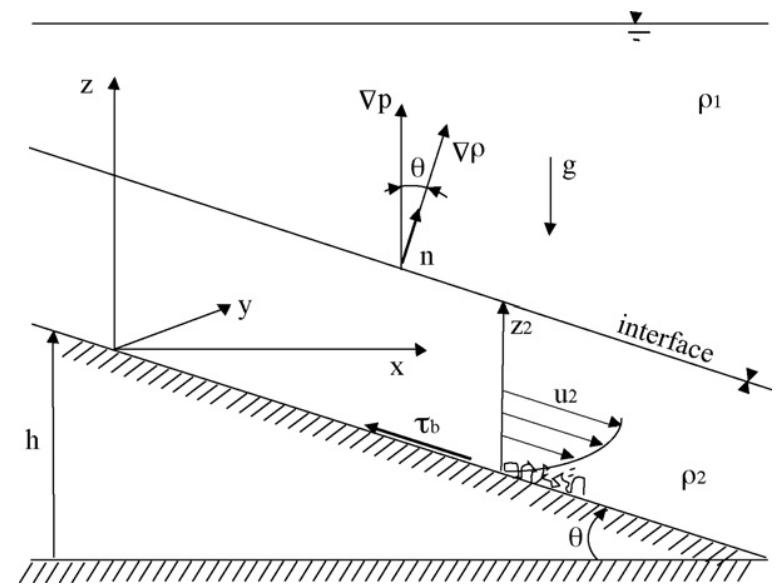

Fig. 5. Definition diagram for vorticity production in a gradually evolving two-layer flow over downslope. 
each contribution in order to relate the bottom generated turbulence with the turbulence produced by the density and velocity gradients. The starting point for the estimation of these contributions is the vorticity equation Baines (1995)

$$
\frac{\mathrm{D} \zeta}{\mathrm{D} t}=\zeta \cdot \nabla \mathbf{u}+\frac{\nabla \rho \times \nabla p}{\rho^{2}}+\nu \Delta \zeta,
$$

where $\mathrm{D} / \mathrm{D} t$ denotes the Lagrangian derivative, $\mathbf{u}=(u, v, w)$ is the velocity vector, $\zeta=\nabla \times \mathbf{u}$ the vorticity vector, $\rho(x, y, z)$ the density, $p(x, y, z)$ the pressure, $v$ the kinematic viscosity, $\triangle$ the Laplacian operator.

The vorticity production related to the enhanced bottom roughness can be included in Eq. (1) through the boundary conditions and can be expressed in the form of the viscous term $v \triangle \zeta$. To model the friction source of vorticity it is assumed that the friction arises essentially due to tangential (shearing) forces and that vertical gradients are much larger than horizontal. In fact, the effects related to horizontal gradients are more important for the processes occurring at the interface between the two layers and these are taken into account with the dimensional analysis of the term $\zeta \nabla u$. Thus, the boundary layer assumptions can be used without any loss of generality, namely that the horizontal gradients are much smaller than the vertical $(\partial / \partial x \ll \partial / \partial z)$ and that the vertical velocity is small compared to the streamwise velocity (i.e. $w \ll u$ ). Moreover, the transverse velocity $v$ and the transverse velocity gradients $\partial / \partial y$ can be assumed negligible as the flow can be treated as two-dimensional in first approximation.

As a starting point to model the bottom friction, the bottom shear stress $\tau_{\mathrm{b}}$ can be written as follows

$$
\frac{\tau_{\mathrm{b}}}{\rho_{2}}=\frac{f_{\mathrm{w}}}{8} u^{2}=v \frac{\partial u}{\partial z},
$$

where $\rho_{2}$ is the density of the lower layer fluid, $u_{2}$ is the velocity in the lower layer, $\partial u / \partial z$ is the streamwise velocity gradient due to the bottom generated shear stress. The relation $\sqrt{\tau_{\mathrm{b}} / \rho}=$ $u \sqrt{f_{\mathrm{w}} / 8}$ has been used, where $f_{\mathrm{w}}=4 c_{\mathrm{f}}$, with $c_{\mathrm{f}}$ being a quadratic-law friction coefficient. To introduce this term into Eq. (1) the Laplacian operator has to be applied on both hand sides of Eq. (2), leaving to

$$
v \Delta\left(\frac{\partial u}{\partial z}\right) \sim \nu \Delta \zeta \approx \frac{f_{\mathrm{w}}}{4}\left[\left(\frac{\partial u}{\partial z}\right)^{2}+\frac{\partial^{2} u}{\partial z^{2}} u\right],
$$

where the streamwise and cross-stream variation of the velocity $u$ has been neglected, since $\partial / \partial y<\partial / \partial x \ll \partial / \partial z$. The viscous term in Eq. (1) can thus be replaced with the right hand side of Eqs. (1) and (3) reads finally as follows

$$
\frac{\mathrm{D} \zeta}{\mathrm{D} t}=\zeta \cdot \nabla \mathbf{u}+\frac{\nabla \rho \times \nabla p}{\rho^{2}}+\frac{f_{\mathrm{w}}}{4}\left[\left(\frac{\partial u}{\partial z}\right)^{2}+\frac{\partial^{2} u}{\partial z^{2}} u\right] .
$$

It is noted that by integrating the last term on the right hand side of Eq. (4) in the $z$ direction, the bottom boundary condition reduces to Eq. (2). We recall that Eqs. (2) and (3) are only a model which is defined in order to include the bottom friction term in the vorticity Eq. (1) and are not used to derive analytically new equations: Eq. (4) will be used below to perform an order of magnitude analysis of the single terms.

In the following, the different production terms on the right hand side of Eq. (4) are scaled separately. 
The generation of $y$-vorticity due to vortex stretching is given by:

$$
(\zeta \cdot \nabla \mathbf{u})_{y}=\left(\frac{\partial u}{\partial z}-\frac{\partial w}{\partial x}\right) \frac{\partial v}{\partial y} .
$$

Using the continuity equation $\nabla \cdot \mathbf{u}=0$ and applying the boundary layer assumptions Eq. (5) gives

$$
(\zeta \cdot \nabla \mathbf{u})_{y}=\left(\frac{\partial u}{\partial z}-\frac{\partial w}{\partial x}\right)\left(-\frac{\partial u}{\partial x}-\frac{\partial w}{\partial z}\right) \sim-\frac{\partial u}{\partial z} \frac{\partial w}{\partial z} .
$$

To evaluate the term $\partial w / \partial z$ we note that the variation of the velocity $w$ in the vertical direction is expected to be related to the acceleration down the slope, i.e. $\partial w / \partial z \propto u_{2} / z_{2} \mathrm{~d}\left(z_{2}+h\right) / \mathrm{d} x$ (see Fig. 5). The scaling arguments for the estimation of the vortex stretching term are chosen under the assumption that the related vorticity production is mainly caused by the velocity shear at the interface. Taking the vorticity thickness $\delta_{v}$ (a more precise definition of $\delta_{v}$ will be given in Section 6.1) as vertical scale and the velocity difference between the two layers $\Delta U$ as velocity scale to estimate the vertical velocity gradient $\partial u / \partial z$, the vortex stretching term is finally approximated as follows

$$
(\zeta \cdot \nabla \mathbf{u})_{y} \sim \frac{\partial u}{\partial z} \frac{u_{2}}{z_{2}} \frac{\mathrm{d}\left(z_{2}+h\right)}{\mathrm{d} x} \approx \frac{\Delta U u_{2}}{\delta_{v}} \frac{1}{z_{2}} \sin \theta .
$$

where $\theta$ denotes the angle of inclination of the slope with respect to the longitudinal direction $x$. Thus, the contribution to the vorticity due to vortex stretching is a result of both the vertical gradient of the streamwise velocity and the acceleration down the slope. We note that Eq. (7) has been written under the assumption that the interface is approximately parallel to the sill slope, $\mathrm{d}\left(z_{2}+h\right) / \mathrm{d} x \approx \sin \theta$ (see Fig. 5).

The baroclinic production of $y$-vorticity reads

$$
\frac{\nabla \rho \times \nabla p}{\rho^{2}}=\frac{1}{\rho^{2}}\left(\frac{\partial \rho}{\partial x} \frac{\partial p}{\partial z}-\frac{\partial \rho}{\partial z} \frac{\partial p}{\partial x}\right) .
$$

Assuming the horizontal pressure gradient is negligible and using the Boussinesq approximation for small density variations (Pawlak and Armi, 1998) it follows

$$
\frac{\nabla \rho \times \nabla p}{\rho^{2}} \sim \frac{g}{\bar{\rho}} \frac{\partial \rho}{\partial x} \sim \frac{g}{\bar{\rho}} \frac{\partial \rho}{\partial n} \sin \theta,
$$

with $\bar{\rho}$ being the mean density between the two layers. The relation $\partial \rho / \partial x=\partial \rho / \partial n \sin \theta$ derives from the flow geometry, in which $n$ is the direction of density gradient (see Fig. 5). For dimensional analysis the vertical length scale $\delta_{\rho}$ represents the shear layer thickness and Eq. (9) can be approximated as follows

$$
\frac{\nabla \rho \times \nabla p}{\rho^{2}} \approx \frac{g^{\prime}}{\delta_{\rho}} \sin \theta
$$

Taking as velocity scale the velocity in the lower layer $u_{2}$, the contribution of $y$-vorticity production due to the bottom roughness can be evaluated as

$$
\frac{f_{\mathrm{w}}}{4}\left[\left(\frac{\partial u}{\partial z}\right)^{2}+\frac{\partial^{2} u}{\partial z^{2}} u\right] \sim \frac{f_{\mathrm{w}}}{4}\left[\left(\frac{u_{2}}{\delta_{\mathrm{b}}}\right)^{2}+\frac{1}{\delta_{\mathrm{b}}} \frac{u_{2}}{\delta_{\mathrm{b}}} u_{2}\right],
$$


where $\delta_{\mathrm{b}}=z_{2} / 2$ is assumed to be the vertical scale for the bottom velocity gradient, with $z_{2}$ and $u_{2}$ being the water depth and velocity in the lower layer, respectively (cf. Fig. 5). The vertical gradient $\partial u / \partial z$ is here scaled using the velocity scale $u_{2}$ and the vertical length scale $\delta_{\mathrm{b}}$, as for the friction production of vorticity the vertical velocity gradient is expected to be mainly caused by the shear generated by friction.

Assuming that the vorticity and density gradients are constant across the interfaces, integrating Eqs. (10) and (11) to obtain the total vorticity produced at a given $x$-location, amounts to multiplying by $\delta_{\nu}, \delta_{\rho}$ and $\delta_{\mathrm{b}}$, respectively. The following interaction parameter can be defined

$$
\begin{aligned}
& \frac{\text { Baroclinic production }}{\text { Vortex stretching }}=\mathcal{Z}_{v} \equiv \frac{\left|\delta_{\rho}\left(\nabla \rho \times \nabla p / \rho^{2}\right)_{y}\right|}{\left|\delta_{v}(\zeta \cdot \nabla \mathbf{u})_{y}\right|} \approx F^{-2}\left(\frac{u_{2}}{\Delta U}\right), \\
& \frac{\text { Friction production }}{\text { Baroclinic production }}=\mathcal{Z}_{r} \equiv \frac{\left|\delta_{\mathrm{b}}\left(\left(f_{\mathrm{w}} / 8\right) \triangle\left(u^{2}\right)\right)\right|}{\left|\delta_{\rho}\left(\nabla \rho \times \nabla p / \rho^{2}\right)_{y}\right|} \approx F_{\ell}^{2}(\sin \theta)^{-1}, \\
& \frac{\text { Friction production }}{\text { Vortex stretching }}=\mathcal{Z}_{r v} \equiv \frac{\left|\delta_{\mathrm{b}}\left(\left(f_{\mathrm{w}} / 8\right) \triangle\left(u^{2}\right)\right)\right|}{\left|\delta_{\nu}(\zeta \cdot \nabla \mathbf{u})_{y}\right|} \approx \frac{z_{2}}{\ell \sin \theta}\left(\frac{u_{2}}{\Delta U}\right),
\end{aligned}
$$

where we recognize that $F^{2}=\left(u_{2}\right)^{2} /\left(g^{\prime} z_{2}\right)$ is the single layer Froude number. The length scale $\ell=2 \delta_{\mathrm{b}} / f_{\mathrm{w}}$ represents a "roughness scale" and $F_{\ell}^{2}=\left(u_{2}\right)^{2} /\left(g^{\prime} \ell\right)$ is a "frictional Froude number", which can be reinterpreted as a measure of the limiting effects of buoyancy on the velocity acceleration in the lower layer, hindered by friction.

Eq. (4) shows that the vorticity related to vortex stretching is balanced by the stabilizing vorticity contribution related to the stratification and to bottom friction. Moreover, while the vorticity generated by the stratification as well as by the vortex stretching are "intrinsic" contributions related directly to the fluid properties, the term resulting from the bottom friction represents an "external" source of vorticity and is built from the boundary conditions. In this sense, the interaction parameters containing the relative contribution of "intrinsic" and the "external" vorticity source are the most interesting parameters. Because there are three parameters defined using three vorticity contributions, they are related to each other and one of them is redundant, i.e. $\mathcal{Z}_{v}=\mathcal{Z}_{r v} / \mathcal{Z}_{r}$.

The vorticity parameters $\mathcal{Z}_{r}$ and $\mathcal{Z}_{r v}$ are plotted in Fig. 6(a) and (b), respectively.

In the smooth case the only restoring force is given by buoyancy, while in the rough cases the gap between frictional and baroclinic production is smaller and they both work for stabilization: this explains why in Fig. 6(a) the curves become nearly horizontal in the rough cases earlier compared to the smooth case.

The parameter $\mathcal{Z}_{r v}$ is plotted in Fig. 6(b) and it shows to increase very fast for $(-0.2<x / L<0)$ for the smooth experiment and for $(-0.2<x / L<0.2)$ and then the growth decreases. This change in the slope of the curves is given by the effects of the acceleration down the sill, so that right to the sill crest, vortex stretching increases faster than the friction production of vorticity. Moving downstream the curves become almost horizontal for the two rough experiments as the friction contribution becomes more important, while for the smooth case a growth is still present.

\section{Estimation of roughness coefficients}

In this section, results from the PIV measurements are used to estimate the effect of friction. The vertical and horizontal length scales are given by the total water depth $H$ and the sill length 

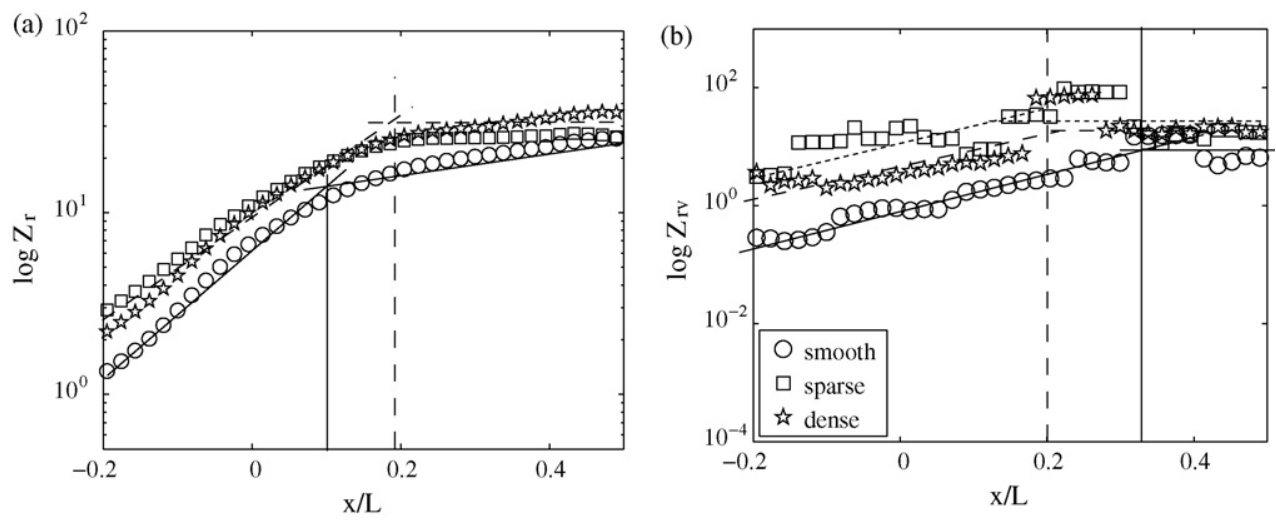

Fig. 6. Vorticity parameters expressing the ratio between the three main contribution of vorticity production in a two-layer stratified flow down a slope with enhanced bottom roughness. (a) $\mathcal{Z}_{r}$ as ratio between the friction and the baroclinic sources and (b) $\mathcal{Z}_{r v}$ as ratio between the friction and the vortex stretching sources vs. $x$ (cf. Eqs. (12b) and (12c)).

$L$ on the right hand side of the sill crest, respectively. As the interfacial instabilities are primarily dominated by the unsteady barotropic flow component and scale with the total water depth, we choose as velocity scale $\sqrt{g^{\prime} H}$.

The bottom roughness coefficients were estimated using the measured flow rates in the upper layer for all three experiments at positions near the sill crest, where the entrainment between the two layers can be neglected (Morin et al., 2004). We expect the flow rates to be decreased in the rough cases, as the bottom roughness effectively results in an increased height of the sill. The normalized flow rate in the upper layer of the rough experiments was thus compared to that of the smooth experiment to obtain the ratio $q_{c, \mathrm{r}} / q_{c, \mathrm{~s}}$, where $q_{c, \mathrm{r}}$ and $q_{c, \mathrm{~s}}$ are, respectively, the flow rate in the upper layer at the sill crest for the rough (index r) and the smooth (index s) experiments. The ratio gives a value of $97 \%$ for the sparse roughness case and $93 \%$ for the dense roughness case as shown in Fig. 7, a value averaged between $-0.05<x / L<0.05$. This average has been done because it is well known that the bottom friction, as well as the interfacial friction, cause the internal hydraulic controls to be shifted from the theoretical positions at the sill crest and the

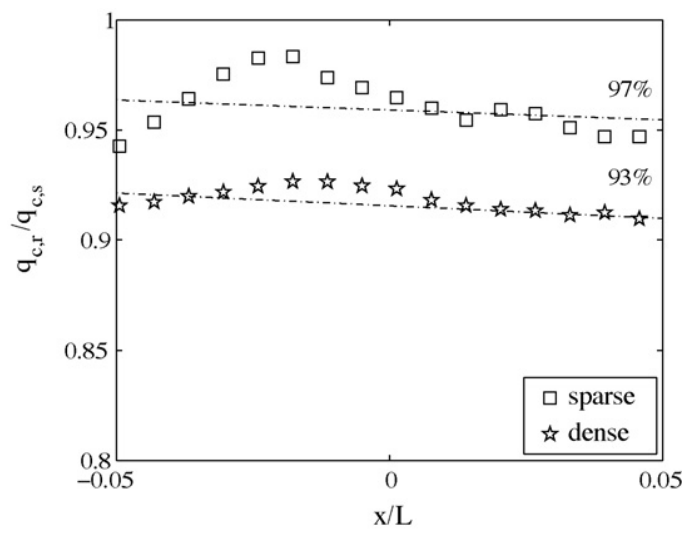

Fig. 7. Normalized flow rate for the rough experiments compared to that of the smooth experiment. 
channel exit. However, the determination of this location is not easy because of entrainment and friction effects (Pratt, 1987; Gu and Lawrence, 2005) as well as due to streamline curvature (Zhu and Lawrence, 1998) and so is omitted within the scope of this study.

An equivalent sill height can be estimated with the help of internal hydraulic theory. Our flow is controlled by two hydraulic control located at the sill crest and the channel exit (Armi and Farmer, 1986). We adapt the control curve in Fig. 3 in Zhu (2002) for the sill crest control by changing the value of $h_{\mathrm{s}}$ to obtain the above estimated ratios $q_{1 c, \mathrm{r}} / q_{1 c \text {, }}$ for the critical flow rate (correspondent to the intersection between the curve of the sill crest control and the channel exit control). The so obtained equivalent sill heights are $h^{\prime}=0.510$ and $h^{\prime}=0.517$ for the sparse and dense roughness configurations, respectively. With the water depth of $40 \mathrm{~cm}$ used in all the performed experiments, this corresponds to heights of $20.4 \mathrm{~cm}$ and $20.7 \mathrm{~cm}$ for the sparse and dense configuration, respectively (see Table 1).

The internal hydraulic energy was defined as $E=h+z_{2}+q^{2} / g^{\prime}\left(1 / z_{2}^{2}-1 / z_{1}^{2}\right)$ and calculated using the measured flow rate and interface position and is plotted in Fig. 8, with the continuous line representing the inviscid hydrostatic prediction of $E / H=0.75$, which can be calculated following the method outlined in Zhu (2002). While the internal hydraulic predictions are in
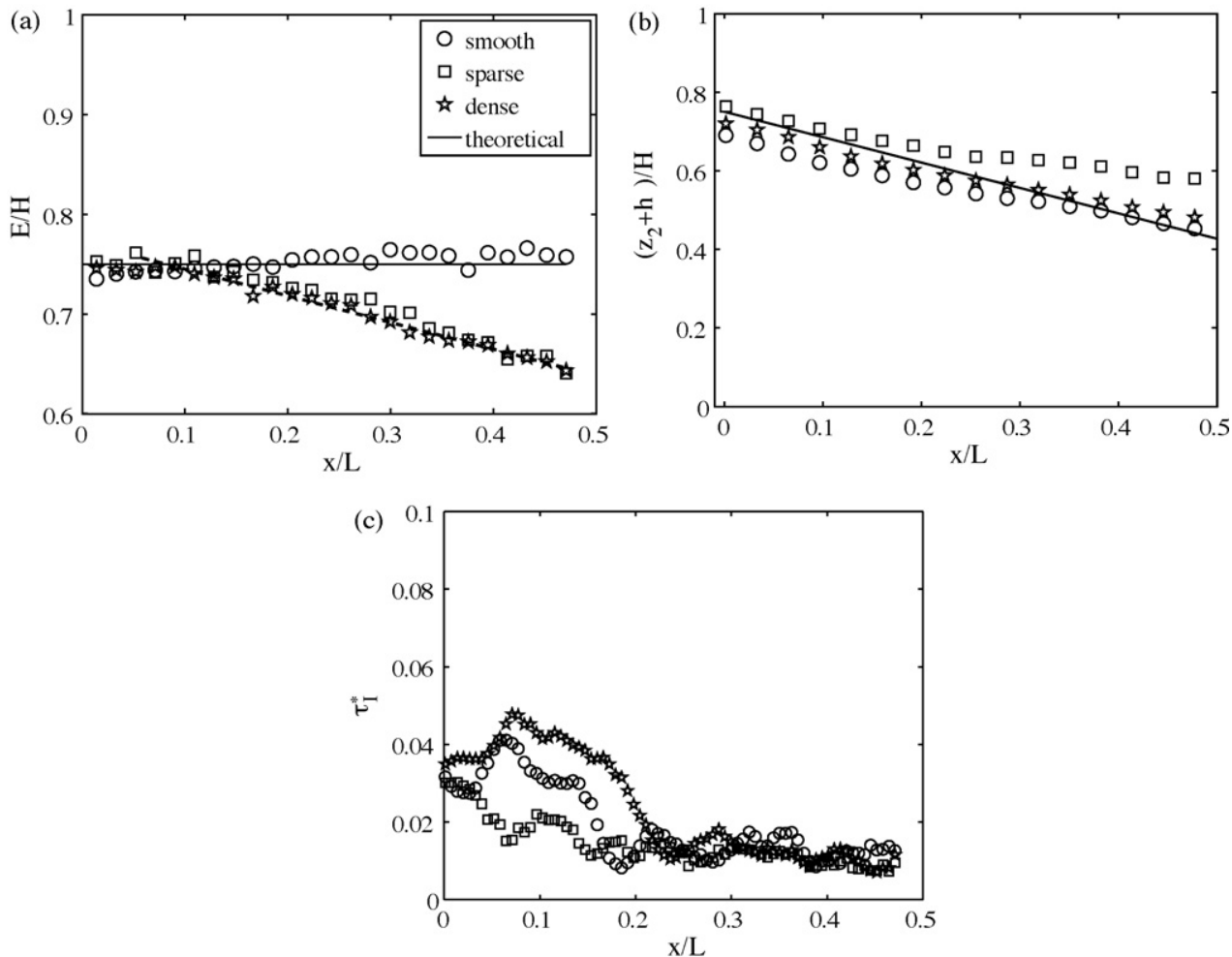

Fig. 8. Comparison between experimental data (symbols) and theoretical prediction using the hydraulic inviscid hydrostatic prediction (continuous line) (a and b) and experimental interfacial friction (c). (a) Total internal hydraulic energy $E$ vs. $x$, (b) mean interface positions calculated as $z_{2}+h$ where $z_{2}=\left(\left.z\right|_{\bar{u}=0.15}-\left.z\right|_{\bar{u}=0.85}\right) / 2$ and (c) dimensionless interfacial friction $\tau_{\mathrm{I}}^{*}=\sqrt{\bar{\rho} \overline{u^{\prime} w^{\prime}}}$ estimated using the measured mean values for $\overline{u^{\prime} w^{\prime}}$ at the interface positions and using the mean measured densities in the lower layer $\bar{\rho}$, normalized with $\Delta \rho g H$. 
very good agreement for experiment 1 , it overestimates the internal energy for experiments 2 and 3 on the right of the sill crest, from $x / L=0.1$ onward. In these experiments internal energy is obviously dissipated due to frictional effects and this is the reason for the poor matching between experimental data and theoretical predictions. Zhu and Lawrence (2000) gave a relation to estimate the energy loss in longitudinal direction due to wall and interfacial friction. Considering only the contribution given by the bottom boundary, an estimation of its friction coefficient can be made using the rearranged relation given in Zhu and Lawrence (2000):

$$
f_{\mathrm{w}}=\frac{2}{u_{2}^{2}} z_{2}\left[\frac{\mathrm{d}(E / H)}{\mathrm{d} x} \frac{H}{L_{\mathrm{c}}}-\tau_{\mathrm{I}}^{*} \frac{\left(z_{1}+z_{2}\right)}{z_{1} z_{2}}\right]
$$

where $(\mathrm{d} E / H) / \mathrm{d} x$ is the measured slope of the energy decrease in longitudinal direction (cf. Fig. 8), $L_{\mathrm{c}}$ is the length of the connecting channel (cf. Fig. 1(a)) and $\tau_{\mathrm{I}}^{*}=-\bar{\rho} \overline{u^{\prime} w^{\prime}}$ is the measured interfacial shear stress between the two layers normalized with $\Delta \rho g H$, calculated using the measured values for $\overline{u^{\prime} w^{\prime}}$ (averaged over time) at the interface positions and using the mean measured densities in the lower layer $\bar{\rho}$. The water depths $z_{1}$ and $z_{2}$ are normalized with the water depth $H$ and the velocity $u_{2}$ with the velocity scale $\sqrt{g^{\prime} H}$. The interfacial shear stress along the longitudinal direction is plotted in Fig. 8(c) showing the largest values for the dense roughness case, followed by the smooth case and finally the sparse roughness case. The so obtained estimated friction coefficients are $f_{\mathrm{w}}=0.024$ and $f_{\mathrm{w}}=0.018$ for the sparse and dense roughness configuration, respectively.

At first glance this seems to be in contradiction with the estimated equivalent sill heights: in the dense roughness case a higher equivalent sill height $h^{\prime}$ is calculated, however resulting in a reduced friction coefficient $f_{\mathrm{w}}$. This is principally due to the fact that in the dense roughness case the interfacial shear stress $\tau_{\mathrm{I}}^{*}$ is larger as compared to the sparse roughness case (cf. Fig. 8(c)) because the interaction between the bottom boundary layer and the interface is considerably larger in the dense roughness case compared to the sparse case. On the other hand, the depth of the lower layer $z_{2}$ is reduced in the dense case as compared to the sparse roughness case and thus also the flow rate. All these effects result in reduced bottom roughness coefficients (cf. Eq. 13) and flow rates for the dense roughness case. This demonstrates that in a two-layer flow the magnitude of bottom roughness is also influenced by the interaction between the bottom boundary layer turbulence and that at the interface.

\section{Discussion}

\subsection{Mean flow characteristics}

In Fig. 9, the averaged velocity fields $U=\sqrt{u^{2}+w^{2}}$ (left column) and the Reynolds-stress $\overline{u^{\prime} w^{\prime}}$ distribution (right column) are shown for all three experiments. In the rough cases the velocity difference between the two layers is lower and as bottom roughness inhibits acceleration down the slope. The Reynolds stresses are significantly higher in the two rough experiments in a thinner shear layer. The vorticity and density thicknesses, first mentioned in Section 4, were obtained from the inverse slope of the best-fit line through the normalized velocity profile between the 15 and $85 \%$ values (Pawlak and Armi, 2000):

$$
\delta_{v}=\left(\left.\frac{\overline{\mathrm{d} \bar{u}}}{\mathrm{~d} z}\right|_{\bar{u}=0.15} ^{\bar{u}=0.85}\right)^{-1} \quad \text { and } \quad \delta_{\rho}=\left(\overline{\left.\frac{\mathrm{d} \bar{\rho}}{\mathrm{d} z}\right|_{\bar{\rho}=0.15} ^{\bar{\rho}=0.85}}\right)^{-1},
$$



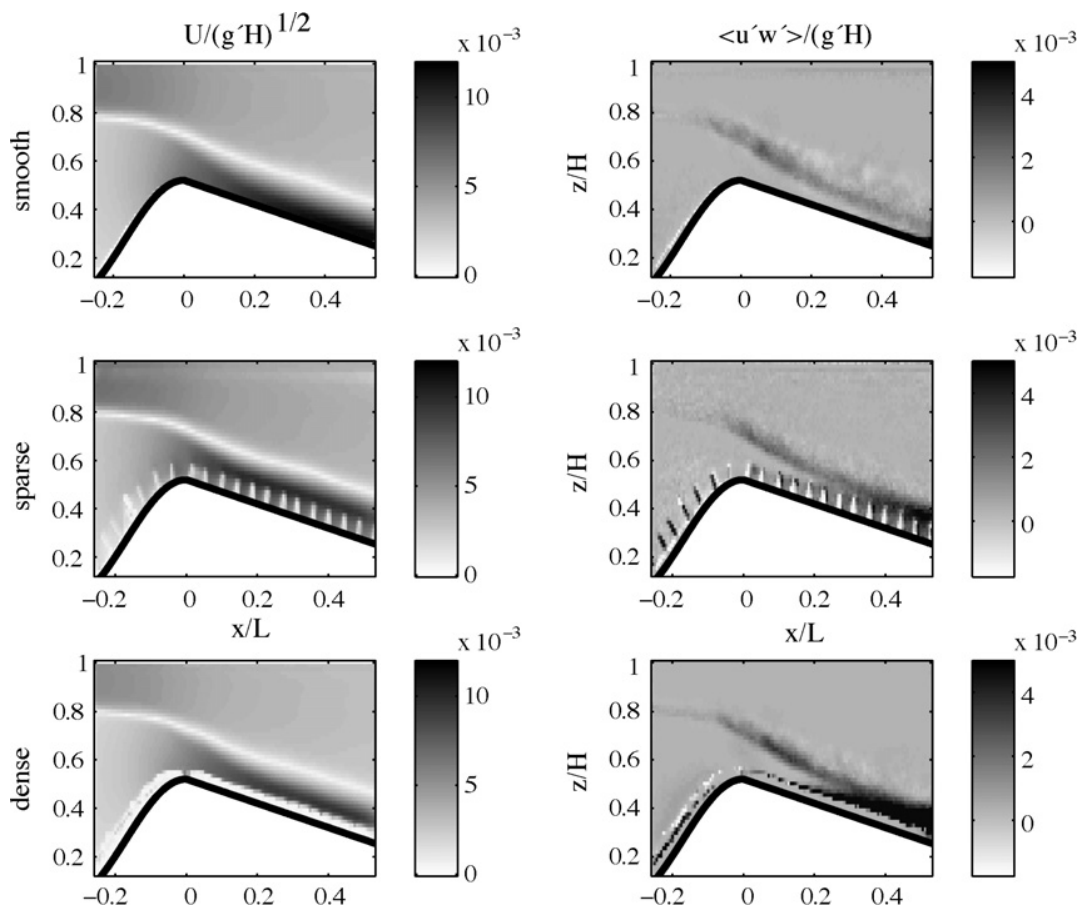

Fig. 9. Mean flow characteristics in the measured flow field. Normalized averaged velocity field $U=\sqrt{u^{2}+w^{2}}$ (left column) and normalized Reynolds stresses $\overline{u^{\prime} w^{\prime}}$ distribution (right column).

respectively, where $\bar{u}$ and $\bar{\rho}$ are the normalized velocity and density, ranging from 0 to 1 . These parameters are plotted for all the experiments in Fig. 10.

The vorticity thickness shows a faster increase in the smooth case (see Fig. 10(a)), followed by the dense roughness case and, finally, the sparse roughness case. The density layer thickness (Fig. 10(b)) is larger right to the sill crest for the smooth case while it shows smaller values for the rough cases. The wake behind the pulsating surge-like currents observed for the
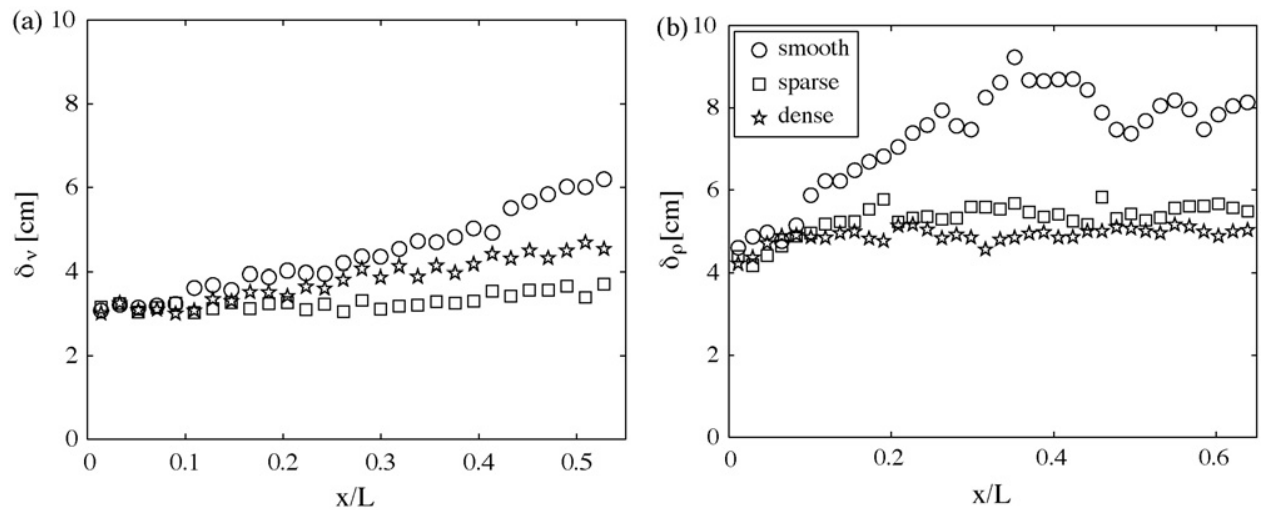

Fig. 10. Time averaged quantities for the performed experiments. (a) Vorticity thickness vs. the longitudinal direction and (b) density layer thickness. 
smooth experiment can not develop right to the sill crest, which results in a reduced increase of the shear and density layer thickness downstream of the sill crest. It is worth noting that in the dense roughness case the growth of the vorticity thickness fits between the other two experiments, while density thickness has the smallest values for this experiment. This can be explained by the fact that in the sparse roughness case the velocity gradients in the velocity shear layer are much larger than those in the dense roughness case thus resulting in reduced values as compared to the dense roughness case. However, density gradients in the density shear layer are larger for the dense roughness case resulting in the smallest values observed in Fig. 10(b).

The configuration with dense roughness elements is more effective in damping the formation of the two-dimensional structures at the interface compared to the sparse configuration. Sparse spacing of coarse, tall roughness elements act almost as single disturbance bodies and the turbulence produced between the elements is not interacting so intensively with the interface as compared to the dense configuration.

Due to the larger height of the roughness elements in the sparse roughness case the growth process of the large-scale surges generated at the interface is disturbed and the collapse of these surges is hindered, so that no wake zone can be observed behind them. In the dense roughness case, the velocity gradients in the lower layer are much stronger than in the sparse roughness case (cf. Fig. 11(a) and (b)) and also the mixing layer is growing faster and becomes larger in the dense roughness case than in the sparse roughness case (cf. Fig. 10): this suggests that the interaction between the boundary layer and the velocity shear interfacial is more intensive for the dense roughness case than for the sparse roughness case. In the sparse roughness case the turbulent bottom layer spreads vertically less rapidly than the two-dimensional structures generated at the interface due to baroclinic/barotropic effects. The effect of the roughness can also be noted on the density layer properties. The normalized concentration profiles are plotted in Fig. 12(a): the concentration profiles become sharper when the roughness increases. This is also demonstrated in Fig. 12(b)-(d), where the concentration at the density interface is compared to the mean concentration in the density layer for the smooth, the sparse and the dense roughness case, respectively. As the roughness increases, the gap between the data increases remarkably. We note that in fact, if one looks at the instantaneous density profiles, they show sharper density
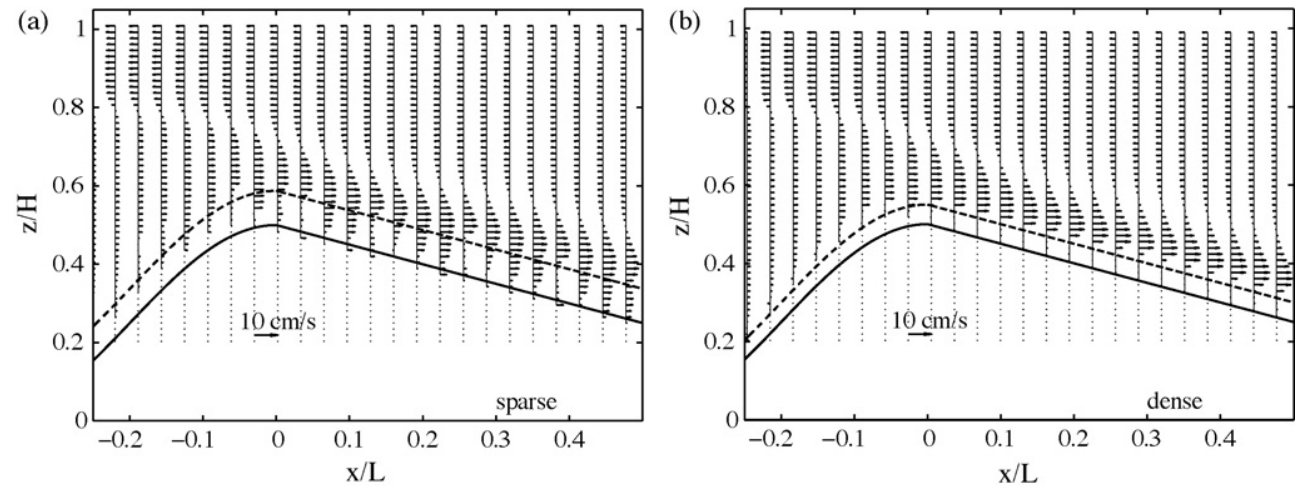

Fig. 11. Measured velocity profiles at different longitudinal sections averaged over 10 barotropic cycles for (a) the sparse roughness case and (b) dense roughness case. The velocity gradients in the lower layer are larger in the dense bottom roughness configuration (b) as in the sparse configuration (a). 

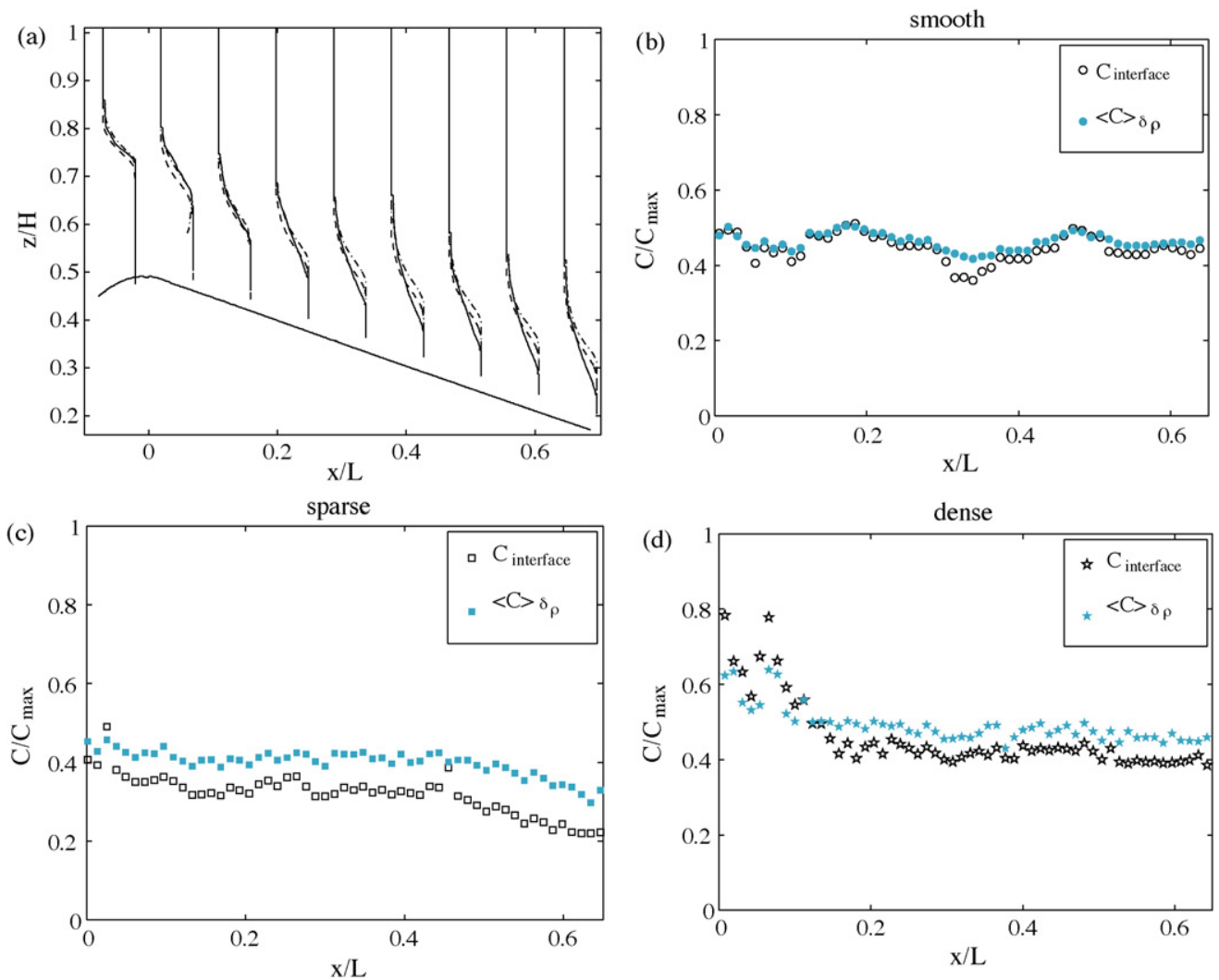

Fig. 12. Concentration characteristics for the performed experiments. (a) Averaged density profiles at different sections: smooth case (continuous line), sparse roughness case (dotted line) and dense roughness case (dashed line). Averaged concentration at the density interface and averaged concentration in the density layer $\delta_{\rho}$ for the smooth case (b), the sparse roughness case (c) and the dense roughness case (d).

interfaces in the smooth case as compared to the rough cases, but in the average the results show the contrary.

In Fig. 13 the vertical profiles of the Richardson numbers

$$
R i=-\frac{g}{\bar{\rho}} \frac{(\partial \rho / \partial z)}{(\partial u / \partial z)^{2}}
$$

are plotted at three different longitudinal sections. The first noticeable difference is that the highest Richardson numbers are observed at higher vertical positions $z / H$ in the smooth case as compared to the bottom roughness cases at all longitudinal sections. This suggests that in the bottom roughness cases the strongest density gradients, or the most unstable regions, are found at lower vertical positions as compared to the smooth case. Also interesting to note is that the thickness of the region with non-zero $R i$ is increasing in the longitudinal direction for the smooth and the dense roughness case, indicating a growing mixed layer, while it decreases in the sparse roughness experiment (cf. Fig. 10). These results are also consistent with those presented in Figs. 12 and 17. The averaged horizontal (left column) and vertical (right column) Reynolds transports, $\overline{u^{\prime} c^{\prime}}$ and $\overline{w^{\prime} c^{\prime}}$, respectively, are plotted in Fig. 14 normalized using the 

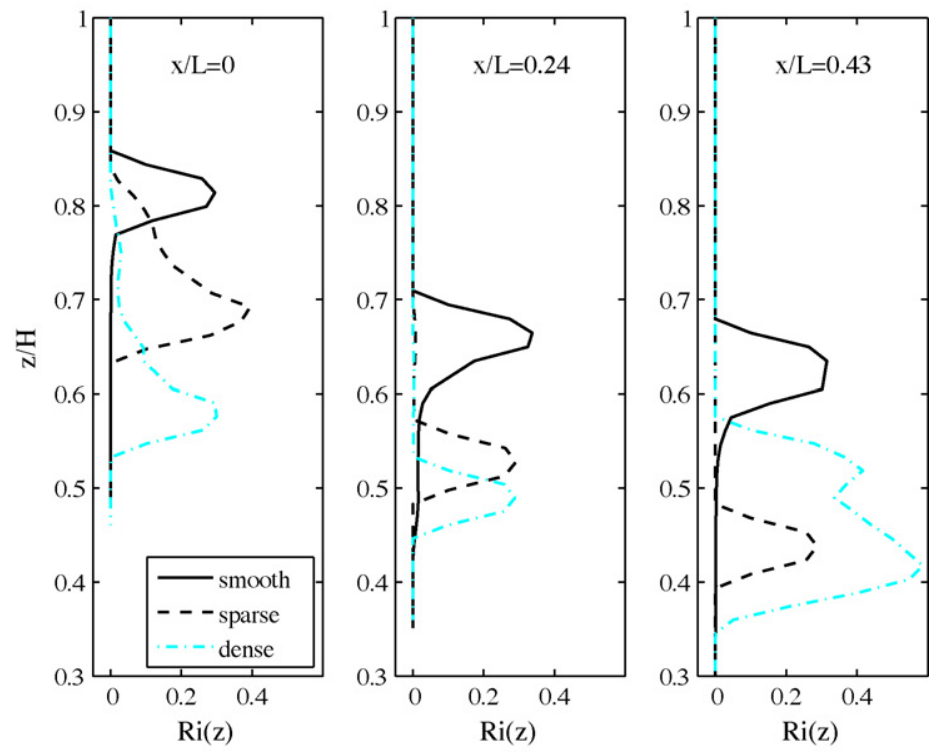

Fig. 13. Vertical distribution of the Richardson number (Eq. (14)) at three different longitudinal sections.
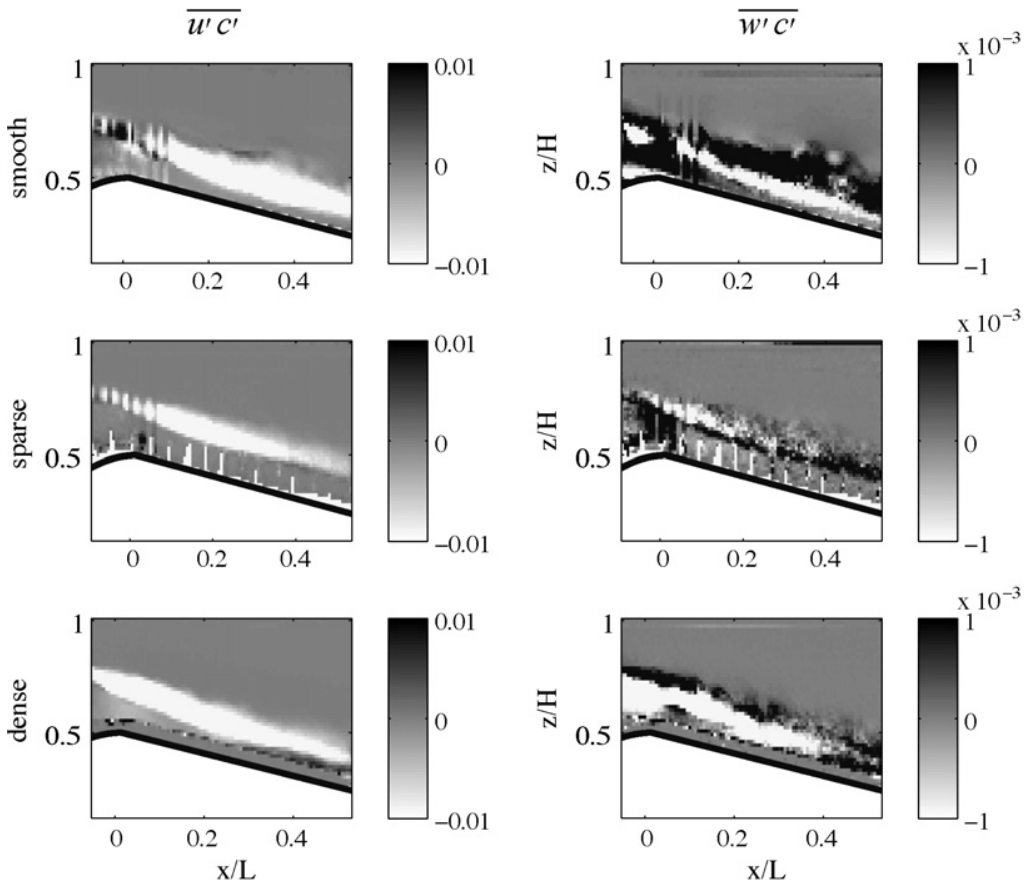

Fig. 14. Buoyant transport $\overline{u^{\prime} c^{\prime}}$ (left column) and $\overline{w^{\prime} c^{\prime}}$ (right column), normalized using the velocity scale $\sqrt{g^{\prime} H}$ and the maximal concentration. 
velocity scale $\sqrt{g^{\prime} H}$ and with the maximal concentration. Positive values represent mass transport from the lower salty layer into the upper fresh water layer, while negative values represent the transport of fresh water into the lower layer. The largest values of the buoyant transport in both directions are observed in the smooth experiment, due to the large concentration fluctuations in the lower layer at the density interface originated by the periodic passing of the large-scale surges. The dense roughness case presents a similar buoyant transport as the smooth case, due to the intensive interaction between the bottom turbulence and the interface. The concentration fluctuations are strongly reduced in the sparse roughness case as the collapse of the surges is hindered, consequently resulting in a reduced turbulent transport. This also explains why the thickness of the region with the highest turbulent transport is much larger in the smooth case as compared to the rough cases. However, bottom roughness causes larger Reynolds stresses at the sheared interface, as demonstrated in Fig. 9, right column. This is also consistent with the results presented in Fig. 10. It is also worthwhile noting that the turbulent transport increases moving to the right of the sill crest for the smooth bottom case, while they are decreasing in the longitudinal direction in the rough cases. In the rough cases the surge structures initially grow, but further downstream the collapsing mechanism and the further growth in the sparse and dense bottom roughness experiments are hindered by friction, thus resulting in reduced concentration fluctuations. This is also consistent with the results presented in Fig. 16(a). These results relative to the turbulent transport distribution are also consistent with the entrainment rates presented in Section 6.3(Fig. 17(a)).

\subsection{The effect of locally enhanced bottom roughness on interfacial large-scale structures}

Fig. 15 shows the interface fluctuation record for a time series of $400 \mathrm{~s}$ duration, constructed from a vertical cut at a fixed location in the spatial image, i.e. at $x / L=0.24$. The vertical fluctuations of the density interface as well as the horizontal size of the large-scale structures are visibly reduced for the rough cases as compared to the smooth case.

Fig. 16(a) shows the standard deviation of the density interface fluctuations shown in Fig. 15 as a measure of the growth rate of the large-scale two-dimensional structures. It is worth noting that while for the smooth experiment there is a constant increase of the standard deviation as
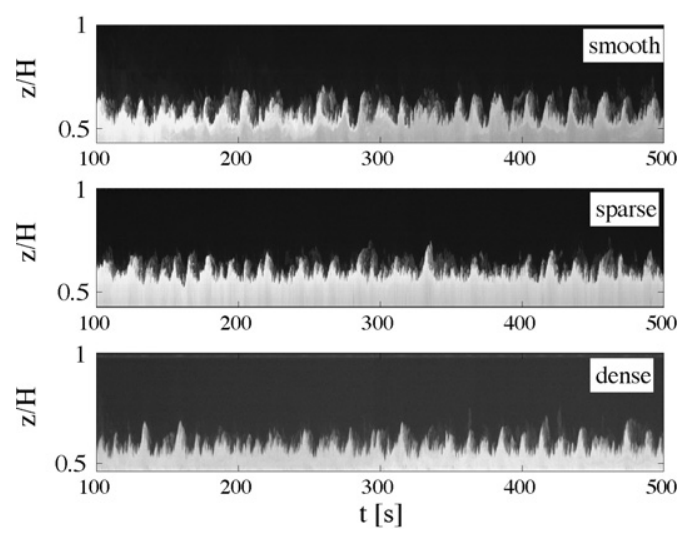

Fig. 15. Interface fluctuation record for a time series of $400 \mathrm{~s}$ duration, constructed from a vertical cut at a fixed location in the spatial image, i.e. at $x / L=0.24$. 

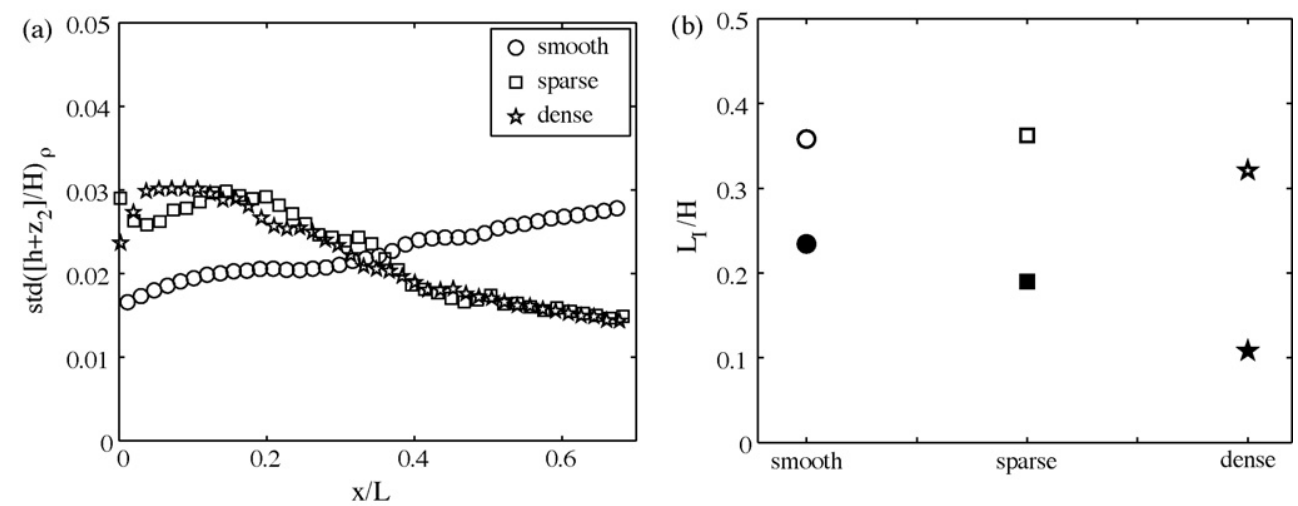

Fig. 16. (a) Standard deviation of the normalized density interface fluctuations; (b) estimated horizontal (empty symbols) and vertical (filled symbols) length scales of the large, two-dimensional structures at the interface.

the flow moves down the slope, the rough experiments show an opposite behavior, with the standard deviation decreasing in longitudinal direction. In the sparse roughness case there is an initial increase in the region $0<x / L<0.15$, and then a continuous decrease can be observed, indicating an initial growth, but a rapid collapse of the large-scale structures at the interface. In the dense roughness case there is a constant decrease, suggesting that no two-dimensional structures are generated at the interface. Moreover, the standard deviation for the rough experiments is higher as compared to that of the smooth case in the initial developing region $x / L<0.3$, while in the growing region of the large scale structures, the fluctuations of the density interface in the smooth case become larger than those of the rough experiments. This is also consistent with the visual observations described above.

Fig. 16(b) shows an estimation of the horizontal (empty symbols) and vertical (filled symbols) length scales of the large, two-dimensional structures at the interface. They were calculated using the time scale $T_{\mathrm{I}}$ obtained from the temporal autocorrelation functions of the horizontal and vertical velocity fluctuations at the interface for $x / L=0.24$, and the mean velocity shear $\Delta U / 2$ as convective velocity scale, e.g. $L_{\mathrm{I}}=T_{\mathrm{I}} \Delta U / 2$. For the dense roughness case, both the horizontal and vertical sizes of the large-scale structures at the interface are reduced, while for the sparse roughness case only the vertical scale is reduced, as compared to the smooth case, and the horizontal scale is roughly the same.

\subsection{The effect of locally enhanced bottom roughness on mixing and entrainment}

Given the velocity field data, estimates of the entrainment coefficient can be obtained. Following Ellison and Turner (1959), the entrainment coefficient is defined as

$$
\alpha_{\mathrm{e}}=\frac{1}{V} \frac{\mathrm{d} q}{\mathrm{~d} x} \quad \text { with } \quad V=\frac{\int u^{2} \mathrm{~d} z}{\int u \mathrm{~d} z}
$$

where $q$ is the flow rate per unit width in the layer of interest and $V$ is a representative velocity for the layer. Here, we also use the relation proposed by Dallimore et al. (2001), where the entrainment 

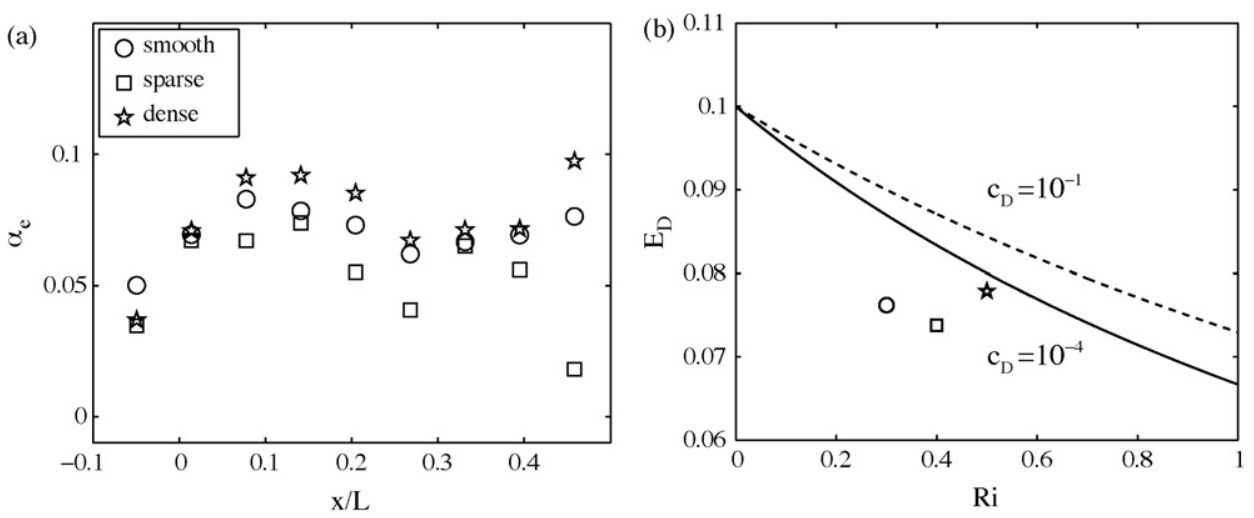

Fig. 17. (a) Entrainment coefficients of the upper-layer fluid along the longitudinal direction and (b) entrainment coefficients compared with the relation proposed by Dallimore et al. (2001) vs. the Richardson number Ri. Symbols represent averaged entrainment coefficients of the performed experiments (smooth case $\circ$, sparse case $\square$, and dense case $\star$ ), while the curves represent Eq. (16) for different bottom drag coefficients $c_{\mathrm{D}}$.

coefficient is defined as follows

$$
E_{\mathrm{D}}=\frac{C_{\mathrm{k}} C_{\mathrm{D}}^{3 / 2}+C_{\mathrm{s}}}{R i+10\left(C_{\mathrm{k}} C_{\mathrm{D}}^{3 / 2}+C_{\mathrm{s}}\right)}
$$

where $C_{\mathrm{k}}$ and $C_{\mathrm{s}}$ are coefficients measuring the efficiency of the boundary-introduced turbulent kinetic energy and of the local shear production, respectively. Past experimental data examined by Sherman et al. (1978) suggested universal values for $C_{\mathrm{k}}=2.2$ and $C_{\mathrm{s}}=0.2$. $R i=g^{\prime} z_{2} / u_{2}^{2}$ is the Richardson number as defined also in Ellison and Turner (1959) and $C_{\mathrm{D}}$ is the bottom drag coefficient. Fig. 17(a) shows the entrainment coefficients for all the performed experiments and the higher values are found in the region $0<x / L<0.15$. The overall higher entrainment coefficients are observed for the dense roughness case, which are of the same order of magnitude of the entrainment coefficients found by Pawlak and Armi (2000), Morin et al. (2004) and Fouli (2006), approaching the value 0.1 . The entrainment coefficients for the smooth case are similar to those observed for the dense roughness case, while the sparse roughness case has the lowest values. This is somehow contradictory, because bottom roughness normally increases the mixing (Dallimore et al., 2001; Fernandez and Imberger, 1999). However, in the flow treated in this study, there are two main sources for mixing between the upper and lower layer: the first one is given by the wakes developed by the large-scale structures periodically generated at the interface and triggered by the barotropic oscillation, while the second one is given by the small-scale, bottom generated turbulence. In the smooth experiment the main source of entrainment is given by the wake region developed in the collapsing phase of the large-scale surges mechanism. In the dense roughness case the main source of entrainment is given by the bottom generated turbulence due to the intensive interaction between the bottom boundary layer and the interface. In the sparse roughness case, the bottom roughness inhibits the collapsing phase of the large-scale barotropic surges and thus, the wake region in which entrainment normally takes place. Moreover, the bottom generated turbulence interacts less intensively with the interface in this case as compared to the dense roughness case. Both these effects result in reduced entrainment coefficients as compared to the other two experiments. These results are also briefly summarized in Table 2. 
Table 2

Summary of the results relative to the large-scale structures growth and collapse mechanisms, the vorticity production and the entrainment

\begin{tabular}{lllll}
\hline Exp. (symbol) & $\begin{array}{l}\text { Growth of } \\
\text { surges }\end{array}$ & $\begin{array}{l}\text { Collapse } \\
\text { of surges }\end{array}$ & $\begin{array}{l}\text { Main source of vorticity } \\
\text { production }\end{array}$ & $\begin{array}{l}\text { Main source of } \\
\text { mixing }\end{array}$ \\
\hline $\begin{array}{l}\text { Smooth } \\
\text { Sparse }\end{array}$ & Yes & Yes & Surges (barotropic) & $\begin{array}{l}\text { Surges (barotropic) } \\
\text { Des }\end{array}$ \\
Yes & No & No & Baroclinic & Surges (barotropic) \\
Bottom roughness
\end{tabular}

In Fig. 17(b), the averaged entrainment quantities reported in all the experiments are plotted (symbols) versus the Richardson number $R i$ as defined in Ellison and Turner (1959). The lines represent the prediction proposed by Dallimore et al. (2001) as in Eq. (16) for different values of the drag coefficient. Increasing the drag coefficient should also increase the entrainment. This is only partially valid for the dense roughness case, as compared to the smooth experiment. For the sparse roughness case the entrainment is lower than in the other two cases, given the same Richardson number. The prediction by Dallimore et al. (2001) overestimates the entrainment coefficients observed here.

\section{Summary and conclusions}

Results of an experimental study on stratified exchange flows down a submerged slope were presented. Three different types of bottom roughness configurations have been used in the experiments, namely a smooth bottom and a sparse and a dense distribution of bottom roughness. By dimensional analysis of the vorticity equation, three parameters have been defined (one of them redundant), in order to analyze the weight of each contribution of vorticity.

Bottom friction coefficients and an equivalent sill height have been estimated through comparison of experimental data with the internal hydraulic theory. Internal energy was found to decrease in the longitudinal direction if bottom roughness is present, while the internal hydraulic theory predictions agree well with the experimental data in the smooth case. The sparse bottom configuration presents the largest bottom friction coefficient $f_{\mathrm{w}}$ however having a smaller equivalent sill height as compared to the dense configuration. This demonstrates that in a two-layer flow the value of the bottom roughness coefficient strongly depends on the interfacial friction which is related to the interaction between the bottom boundary layer and the overlying interface.

The different bottom roughness elements have been shown to behave differently with respect to the interfacial wave activity: enhanced bottom roughness has shown to intensify the interaction between the lower layer and the sheared interface, resulting in hindering the growth (in the dense roughness case) or the collapse (in the sparse roughness case) of large-scale surge-like flows (see Table 2). These behaviors also causes the Reynolds stresses to increase in a thinner sheared interface as bottom roughness increases, while the turbulent transport decreases in the sparse roughness case. The length scales of the large-scale surges has been estimated revealing a reduction of their size if enhanced bottom roughness is present.

Finally, two main sources for mixing have been identified: the first one is given by the wakes developed in the lee of the large-scale structures, while the second one is given by the smallscale, bottom generated turbulence. The entrainment in the smooth experiment is predominantly caused by the wake region developed in the collapsing phase of the large-scale surges. In the dense roughness case, the main source of entrainment is given by the bottom generated turbu- 
lence. In the sparse roughness case the bottom roughness inhibits the collapsing phase of the large-scale structures and thus the wake region in which entrainment normally takes place. Moreover, the interaction between the bottom boundary layer and the interface is weak. Both these behaviors result in reduced entrainment coefficients as compared to the other two configurations (see Table 2).

\section{Acknowledgement}

The authors would like to thank the German Research Foundation (DFG Ji 18/12-1) for its support in conducting this research.

\section{References}

Afanasyev, Y.D., Peltier, W.R., 2001. On breaking internal waves over the sill in Knight Inlet. Proc. R. Soc. 457 (2016), 2799-2825.

Anati, D.A., Assaf, G., Thompson, R.O.R.Y., 1997. Laboratory models of sea straits. J. Fluid Mech. 81, 341-351.

Armi, L., Farmer, D.M., 1986. Maximal two-layer exchange through a contraction with barotropic net flow. J. Fluid Mech. 164, 27-58.

Assaf, G., Hecht, A., 1974. Sea starits: a dynamical model. Deep Sea Res. 21, 947-958.

Baines, W.D., 1995. Topographic Effects in Fluids. Cambridge University Press, Cambridge.

Dallimore, C.J., Imberger, J., Ishikawa, T., 2001. Entrainment and turbulence in saline underflow in Lake Ogawara. J. Hydr. Eng., ASCE 127 (11), 937-948.

Daviero, G.J., Roberts, P.J.W., Maile, K., 2001. Refractive Index Matching in Large-Scale Stratified Experiments. Exp. Fluids 31, 119-126.

Ellison, T.H., Turner, J.S., 1959. Turbulent entrainment in stratified flows. J. Fluid Mech. 6, 423-448.

Farmer, D.M., Armi, L., 1986. Maximal two-layer exchange over a sill and trough a combination of a sill and a contraction with barotropic flow. J. Fluid Mech. 164, 53-76.

Fernandez, R.L., Imberger, J., 1999. Bed roughness induced entrainment in a high Richardson number underflow. J. Hydr. Eng. 44 (6), 725-738.

Fouli, H.R.S., 2006. An experimental study of interfacial waves and instabilities in exchange flows over a smooth sill. Ph.D. Thesis. Edmonton, University of Alberta, Dep. of Civil and Environmental Engineering.

Gu, L., Lawrence, G.A., 2005. Analytical solution for maximal frictional two-layer exchange flow. J. Fluid Mech. 543, $1-17$.

Hansen, B., Osterhus, S., 2000. North Atlantic-Nordic Seas exchanges. Prog. Oceanogr. 45 (2), 109-208.

Hebbert, B., Imberger, J., Loh, I., Patterson, J., 1979. Collie river underflow into the Wellington reservoir. J. Hydr. Div., ASCE 105 (5), 533-545.

Morin, V.M., Zhu, D.Z., Loewen, M.R., 2004. Supercritical exchange flow down a sill. J. Hydr. Eng. 130 (6), $521-531$.

Negretti, M.E., Zhu, D.Z., Jirka, G.H., 2007. Barotropically induced interfacial waves in stratified two-layer flows over a sill. J. Fluid Mech. 592, 135-154.

Og`uz, T., Özsoy, E., Latif, M., Sur, H., Ünlüata, Ü., 1990. Modeling of hydraulically controlled exchange flow in the Bosphorus Strait. J. Phys. Oceanogr. 20, 945-965.

Pawlak, G., Armi, L., 1998. Vortex dynamics in a spatially accelerating shear layer. J. Fluid Mech. 376, 1-35.

Pawlak, G., Armi, L., 2000. Mixing and entrainment in developing stratified currents. J. Fluid Mech. 424, 1-37.

Perry, A., Schofield, W., Joubert, P., 1969. Rough wall turbulent boundary layers. J. Fluid Mech. 37, 383.

Pratt, L.J., 1987. Rotating shocks in a separated laboratory channel flow. J. Phys. Oceanogr. 17, 483-491.

Scinocca, J.F., Peltier, W.R., 1989. Pulsating downslope windstorms. J. Atmos. Sci. 46, 2885-2914.

Sherman, F.S., Imberger, J., Corcos, G.M., 1978. Turbulence and mixing in stably stratified waters. Ann. Rev. Fluid Mech. 10, 267-288.

Sherwin, T.J., Turrell, W.R., 2005. Mixing and advection of a cold water cascade over the Wyville Thomson Ridge. Deep Sea Res. 52, 1392-1413.

Skyllingstad, E.D., Wijesekera, K.D., 2004. Large-eddy simulation of flow over two-dimensional obstacles: high drag states and mixing. J. Phys. Oceanogr. 34, 94-112. 
Thorpe, S.A., 1983. A laboratory study of stratified accelerating shear flow over a rough boundary. J. Fluid Mech. 138, $185-196$.

Zaremba, L.J., Lawrence, G.A., Pieters, R., 2003. Frictional two-layer exchange flow. J. Fluid. Mech. 474, $339-354$.

Zhu, D.Z., Lawrence, G.A., 1998. Non-hydrostatic effects in layered shallow water flows. J. Fluid. Mech. 355, 1-16.

Zhu, D.Z., Lawrence, G.A., 2000. Hydraulics of exchange flows. J. Hydr. Eng., ASCE 126 (12), 921-928.

Zhu, D.Z., 2002. Control curves for two-layer flows. J. Hydr. Eng. 128, 113-116. 